\title{
OPEN Adiabatic mode transformation in width-graded nano-gratings enabling multiwavelength light localization
}

\author{
Moein Shayegannia ${ }^{1}$, Arthur O. Montazeri ${ }^{1,3}$, Katelyn Dixon $^{1}$, Rajiv Prinja ${ }^{1}$, \\ Nastaran Kazemi-Zanjani ${ }^{1}$ \& Nazir P. Kherani ${ }^{1,2} \bowtie$
}

We delineate the four principal surface plasmon polariton coupling and interaction mechanisms in subwavelength gratings, and demonstrate their significant roles in shaping the optical response of plasmonic gratings. Within the framework of width-graded metal-insulator-metal nano-gratings, electromagnetic field confinement and wave guiding result in multiwavelength light localization provided conditions of adiabatic mode transformation are satisfied. The field is enhanced further through fine tuning of the groove-width $(w)$, groove-depth $(L)$ and groove-to-groove-separation (d). By juxtaposing the resonance modes of width-graded and non-graded gratings and defining the adiabaticity condition, we demonstrate the criticality of $w$ and $d$ in achieving adiabatic mode transformation among the grooves. We observe that the resonant wavelength of a graded grating corresponds to the properties of a single groove when the grooves are adiabatically coupled. We show that $L$ plays an important function in defining the span of localized wavelengths. Specifically, we show that multiwavelength resonant modes with intensity enhancement exceeding three orders of magnitude are possible with $w<30 \mathrm{~nm}$ and $300 \mathrm{~nm}<d<900 \mathrm{~nm}$ for a range of fixed values of $L$. This study presents a novel paradigm of deep-subwavelength adiabatically-coupled width-graded gratings-illustrating its versatility in design, hence its viability for applications ranging from surface enhanced Raman spectroscopy to multispectral imaging.

Spectroscopy - the study of light-matter interactions-is usually carried out through the dispersion of scattered light by diffraction gratings. High sensitivity spectroscopy of molecular species has been made possible by surface enhanced Raman scattering and surface enhanced fluorescence microscopy techniques ${ }^{1-4}$. These techniques benefit from resonant light-matter interactions owing to optimal light confinement within structures at nanometer length scales. Various nanostructures, such as nano-hole arrays ${ }^{5,6}$, high aspect ratio nano-grooves ${ }^{7,8}$, gold nano-disks ${ }^{9,10}$, and metal-insulator-metal (MIM) waveguides ${ }^{11,12}$, localize the optical field within subwavelength cavities due to light coupling with its resonant modes. Among these nanostructures, MIM-based nano-gratings offer the distinct advantage of shaping its desired electromagnetic response, while simultaneously enhancing the optical intensity in both the near-field and far-field ${ }^{11,13-15}$

To achieve enhanced localized fields within nano-gratings, optimal energy transfer is required between the incident light, surface plasmon polaritons (SPPs) propagating atop the grating structure, and the SPPs within the grooves. This can be achieved through impedance matching between the incident light and the SPPs (atop and within the grating) and adiabatic transformation of modes in between the grooves of a nano-grating ${ }^{11,16-18}$. In general, adiabaticity is possible in graded nano-gratings or structures that support rainbow trapping ${ }^{11,18}$. Geometric grading of nano-gratings can be either in depth or in width or both depth and width of the groove. Graded gratings provide the necessary multiwavelength enhancement required in certain applications such as single molecule detection in surface enhanced Raman spectroscopy, surface enhanced Fluorescence microscopy, or infrared spectroscopy ${ }^{2}$. Nevertheless, reducing the gap between the two adjacent metallic side-walls of the groove to the nanometer range within a grating intensifies electromagnetic fields within the cavity and in the

${ }^{1}$ Department of Electrical and Computer Engineering, University of Toronto, Toronto, ON M5S 3G4, Canada. ${ }^{2}$ Department of Material Science and Engineering, University of Toronto, Toronto, ON M5S 3E4, Canada. ${ }^{3}$ Lawrence Berkeley National Laboratory, 1 Cyclotron Rd., Berkeley, CA 94720, USA. ${ }^{\square}$ email: kherani@ ecf.utoronto.ca 
near-field above it. Despite the challenges in fabrication of depth-based graded nano-gratings, width-based graded structures can be easily fabricated using planar lithography and thus open a new paradigm for plasmonic nano-gratings with a rich space of design parameters ${ }^{2,19,20}$.

In this paper, we examine the significant role of surface plasmon polariton coupling and interaction mechanisms in subwavelength gratings vis-à-vis shaping the optical response of the grating. We undertake an in-depth parametric investigation of the requisite conditions for adiabaticity in width graded nano-gratings. Specifically, we carry out controlled perturbative variations in depth $(L)$, width $(w)$, and groove-to-groove separation $(d)$ to determine the parameter set of $L, w$ and $d$ that yield adiabatic mode transformation and thus impedance matching within the width graded nano-gratings. We further contrast resonant modes in graded and non-graded gratings and thus provide insight into the cruciality of the geometric parameters $w$ and $d$ in attaining adiabaticity in graded nano-gratings as well as in shaping the electric field profile (intensity, FWHM, and Q-factor).

Here below, following the introduction of the concept of resonance in periodic and graded MIM gratings we delineate the four principal coupling mechanisms that define the resonant condition in graded gratings. Analytically we develop the conditions for adiabaticity in width-graded gratings. Then we present detailed parametric studies of: (1) single nano-groove; (2) non-graded/Uniform nano-gratings; and (3) width-graded nano-gratings.

\section{Results}

By availing the width of nano-grooves as a tunable parameter, we open a new avenue in light trapping within the field of subwavelength chirped gratings. Groove widths of less than $30 \mathrm{~nm}$ become a principle geometric parameter for effective light trapping in MIM gratings. This is due to the coupling of the evanescent plasmonic fields on the side walls of the groove. The schematic of the width-graded nano-grating under study in this article is shown in Fig. 1a where the smallest groove is situated at the center and is surrounded by nano-grooves with increasing groove widths on either side. Figure $1 \mathrm{~b}$ shows a cartoon of multiwavelength light trapping within the width-graded grating, achieved through parametric optimization of phase engineering that results in synergistic SPP trapping and waveguiding effects - the details of which are described in the following sections. For brevity, hereafter we refer to the incident wavelength simply by $\lambda$ and denote the two types of lamellar MIM gratings as either 'uniform gratings' or 'graded gratings'.

Concept of resonant condition. To provide strong electromagnetic field confinement within the nanogrooves of periodic and graded MIM gratings, two conditions must be satisfied ${ }^{21}$. The first, a phase matching condition, which is:

$$
\beta_{j}-\beta_{j \pm 1}=m\left(\frac{2 \pi}{\Lambda_{j}}-\frac{2 \pi}{\Lambda_{j \pm 1}}\right),
$$

where $\beta_{j}$ is the plasmonic propagation mode constant in the $j$ th groove, $m$ is the mode number, and $\wedge$ is the grating period. In a graded grating, the period increases with groove width $\left(w_{j}=w+j \Delta\right)$, that is, $\wedge_{j}=d+w_{j}$. The second condition is the coupling between field amplitudes of the resonant modes slowly moving in adjacent grooves. This is given $b^{21}$ :

$$
d A_{j}=-i \frac{\beta_{j}}{\left|\beta_{j}\right|} C_{j j \pm 1}^{m} A_{j+1(x)} e^{i\left(\beta_{j}-\beta_{j+1}-\frac{m 2 \pi}{\Lambda_{j}}\right) x} d x
$$

where $A_{j}$ represents resonant field amplitude in the $j$ th groove, and $C_{j j \pm 1}^{m}$ is the magnitude of coupling between the $m$ th mode in the $j$ th and $(j+1)$ th groove, which is defined as:

$$
C_{j j \pm 1}^{m}=\frac{\pi}{2 \lambda} \int_{-w_{j} / 2}^{w_{j} / 2} E_{j}^{*} \varepsilon_{j}(x) E_{j+1} d x
$$

where $E_{j}$ is the localized electric field in the $j$ th groove, and $\varepsilon_{j}(x)$ is the perturbed dielectric in groove $j$; for a periodic grating $\varepsilon_{j}$ is independent of $x$ while for a graded grating $\varepsilon_{j}$ is a function of $x$. The boundaries of the integral are $-\frac{w_{j}}{2}$ and $\frac{w_{j}}{2}$ where $w_{j}$ is width of groove $j$.

To satisfy the phase matching condition of Eq. (1) and to attain a strong coupling coefficient per Eqs. (2) and (3), four primary coupling mechanisms are identified between the incident field and the SPPs on the surface and on the sidewalls of a subwavelength grating which contribute to the resonant behavior of the structure. Each of these coupling mechanisms is illustrated in Fig. 1c; for brevity, we show each of these in a single groove yet recognize that any groove might support any number of these mechanisms to localize electromagnetic field within a groove.

Coupling mechanisms. Coupling of incident wave to the cavity mode. An incident plane wave couples to a grating structure through double reflection Fabry-Perot resonator modes. Specifically, the impedance mismatch between the effective refractive index of each groove (local mode index) and free space causes reflection of the coupled wave at the top of the grating. A similar reflection of the wave also occurs at the bottom (metallic surface) of the groove. These give rise to multiple reflections and thus a standing wave ${ }^{22}$. For a very small groove width $(w)$, only the fundamental mode plays a significant role, while all other modes are strongly evanescent in the groove $e^{23}$. This coupling mechanism is illustrated in groove $j=1$ in Fig. $1 c$, and can be described by ${ }^{24}$ : 


\section{b Incident wideband light}

a

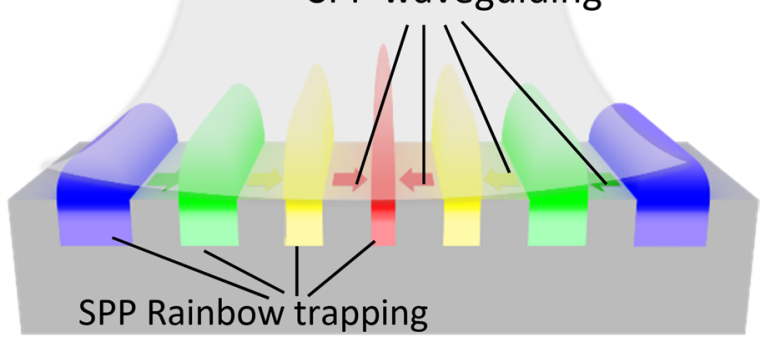

E Incident light

\section{C}
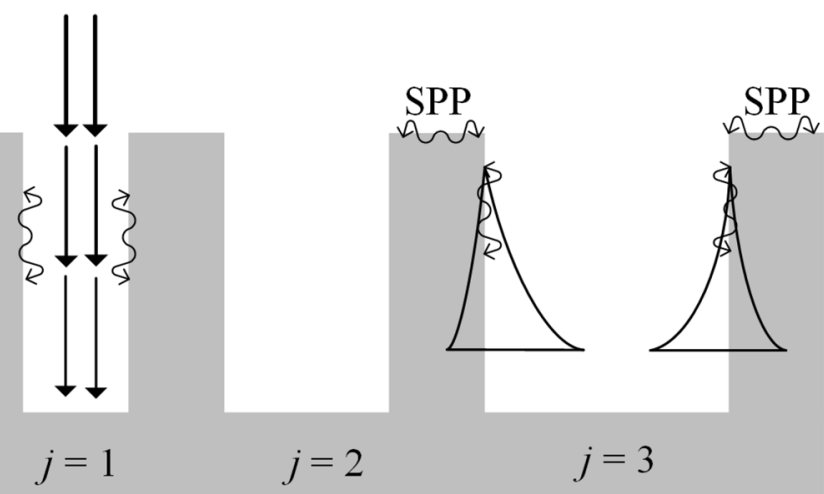

$j=-2$

$j=-1$

$j=0$

$j=1$

$j=3$

Figure 1. (a) Schematic of an MIM-based grating with $W, d, L$ and $\Delta$ representing groove width, groove-togroove separation, groove depth and the width gradient, respectively; $j$ is the groove number. This structure represents a width-graded nano-grating which is symmetric around the centrally situated groove $j=0$. (b) A cartoon illustration of rainbow trapping in the plasmonic width nano-grating; smaller the groove width, larger the wavelength of the localized field. Also, effect of SPPs waveguiding on top of the grating in the direction of increased effective index atop the grating is illustrated by the arrows on the surface, and the increasing intensity of the localized field at smaller groove widths. Gradient in the trapped field intensity shows the location of hotspots within and on top of the grooves. (Not to scale.) (c) Illustrating various coupling mechanisms for mode localization. For brevity, every groove showcases a potential mode coupling mechanism which in general can occur in any other groove. Grooves denoted as $j=-2$ and $j=0$ show the weak and strong coupling between the SPPs excited on the opposing side-walls of the groove, respectively. The evanescent mode coupling from adjacent grooves is shown for groove $j=-1$, specifically coupling with adjoining grooves $j=-2$ and $j=0$. Groove $j=1$ shows the coupling of incident light with the cavity mode. Groove $j=3$ shows coupling of surface waves with the cavity modes and thus exciting SPPs on the groove's inner walls. (Not to scale.). (a, b) were drawn in Community, B. O. (2018). Blender-a 3D modeling and rendering package. Stichting Blender Foundation. Retrieved from http://www.blender.org. (c) was draw in Microsoft Corporation (2016). Microsoft Visio. Retrieved from https://products.office.com/en/visio/flowchart-software.

$$
4 n_{\mathrm{eff}} L \cos \theta=(2 \mathrm{~m}-1) \lambda_{0},
$$

where $n_{e f f}$ is the effective index of the groove, $\theta$ is the angle of incidence, $L$ is the groove depth, $m$ is the mode number, and $\lambda_{0}$ is the incident wavelength.

Coupling of surface waves to a cavity mode. Surface electromagnetic waves or surface plasmon polaritons are transverse magnetic (TM) traveling waves along an interface between two media with opposite signs of the real part of the dielectric constant. A groove carved into a metal film creates a local optical inhomogeneity which makes possible the matching of the in-plane $k$ vectors of the incident wave and the SPPs. The proximal repetition of such grooves - forming a subwavelength grating - guides the incident light along the surface, while forming surface plasmon polaritons. Conservation of momentum dictates that the coupling between the wave vector of light and the wave vector of surface plasmon polaritons occurs with no change in frequency, albeit the wavelength can be compressed by changes in the effective index ${ }^{21}$. SPPs, at the interface of the grating surface and air, travel down the side-walls of the grooves-coupling with the fundamental photonic mode of that groove-and 


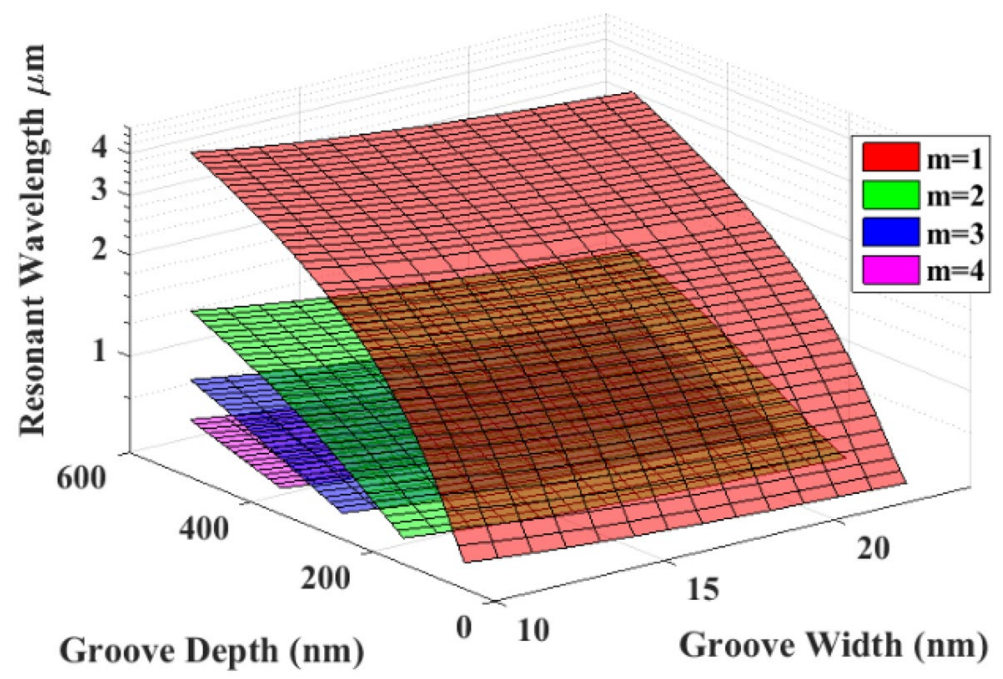

Figure 2. Theoretical relationship between groove depth, groove width, and the resonant wavelength of a single groove. $\mathrm{m}$ represents the mode number of the resonating field within the cavity. Note that the resonant wavelength can be controlled by changing either the groove width or depth.

lead to high electric field intensity inside the grooves. This coupling mechanism is illustrated in groove $j=3$ of Fig. $1 \mathrm{c}$ and is described by ${ }^{22}$ :

$$
\beta_{j}=k \sin \theta \pm m \frac{2 \pi}{\Lambda_{j}}
$$

Intra-groove coupling between the SPPs on the side-walls of an MIM cavity. For very narrow groove widths $(w \lesssim 100 \mathrm{~nm})$ in MIM structures, each groove serves as a modified Fabry-Perot resonator ${ }^{25}$. The SPPs excited on the side-walls of a narrow subwavelength groove, where $w \ll \lambda$ and the effective index is high, couple by virtue of the overlapping evanescent fields. This coupling leads to a significant electric field enhancement within the narrow groove ${ }^{16}$. The effective index is calculated using the following set of equations ${ }^{22}$ :

$$
\begin{gathered}
\tanh _{1} \frac{\mathrm{w}}{2}=-\frac{\mathrm{k}_{2} \varepsilon_{1}}{\mathrm{k}_{1} \varepsilon_{2}}, \\
k_{i}^{2}=\beta_{j}^{2}-k_{0}^{2} \varepsilon_{i}, i=1,2, \\
\beta_{j}=n_{\mathrm{eff} j} k_{0},
\end{gathered}
$$

where $k_{1}$ and $k_{2}$ represent the wavevector of light in the metal and insulator in the MIM cavities, respectively. In contrast, in the limit of wide grooves $(w \approx \lambda \text { or } w \geq 100 \mathrm{~nm})^{25}$, the dispersion relation of the SPPs on the side-walls of the groove is similar to that of a semi-infinite dielectric-metal interface ${ }^{22}$. These coupling mechanisms are illustrated in grooves $j=0$ (intra-groove coupling of side-wall SPPs) and $j=3$ (weak or no coupling of side-wall SPPs), respectively, in Fig. 1c.

The theoretical dependence of the resonant wavelength of a single groove on its width $(w)$ and depth $(L)$, as described by Eqs. (4-8), is illustrated in Fig. 2.

Inter-groove coupling between evanescent fields of adjacent grooves. In grooves $j=-2,-1$, and 0 of Fig. $1 c$, the localized electromagnetic field penetrates the inter-groove metal and thus couples to the adjacent groove modes. The inter-groove penetration of these fields is caused by the finite conductivity of the metal in the visible and near infrared region, and it occurs if the penetration depth of these evanescent SPPs $\left(\delta_{\text {spp }}\right)$ is comparable to the separation between the grooves $(d)^{17}$. Two asymptotic regimes are considered in this case: the optical regime (near/mid infrared) and the electrostatic regime (visible wavelength). In the optical regime, $\delta_{\text {spp }} \approx \delta_{\text {metal }}$, whereas in the electrostatic region, $\delta_{\text {spp }} \ll \delta_{\text {metal }}$, where $\delta_{\text {metal }}$ is the skin depth of the metal ${ }^{17}$. Groove width and excitation wavelength determine the crossover point between these two regimes. These parameters and the separation between adjacent grooves determine whether an evanescent field from one groove can couple to that in an adjacent groove.

Concept of adiabatic mode transformation. To achieve multiwavelength localization in a graded grating, perturbation in the dielectric properties needs to occur sufficiently slowly so as to allow an adiabatic process 


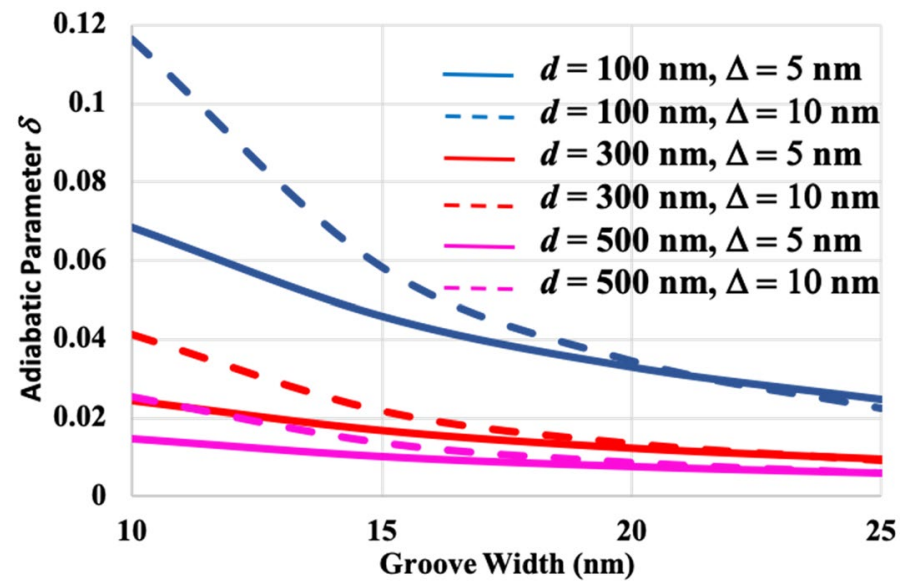

Figure 3. Theoretical relationship between the adiabatic parameter $(\delta)$ and groove width $(w)$ as a function of groove-to-groove separation $(d)$, and gradient in groove width $(\Delta)$. Note that higher values of $d$ and lower values of $\Delta$ lead to smaller values of $\delta$. The smaller the $\delta$, the better the adiabatic mode transformation between the nano-grooves.

whereby dissipation of the EM energy is minimized. Under adiabatic mode transformation, the graded grating is impedance-matched; that is, each groove resonates strongly at a single wavelength while transferring non-resonant modes to an adjacent groove(s) (for example, see the cartoon in Figs. 1b, 5 and 6). The adiabatic parameter $\delta$ is derived using the Wentzel-Kramers-Brillouin approximation ${ }^{26}$ and by simplifying Schrodinger's equation for a one-dimensional motion of a single particle in a "quasi-classical" system. This approximation necessitates ${ }^{27}$ :

$$
\left|\frac{\hbar \sigma^{\prime \prime}(x)}{\left(\sigma^{\prime}(x)\right)^{2}}\right| \ll 1
$$

where $\hbar$ is the reduced Planck's constant, $\sigma^{\prime}(x)$ and $\sigma^{\prime \prime}(x)$ are the first and second derivatives of the $\sigma(x)$, the phase in the solution of Schrodinger's equation wave function approximated by $\sigma(x) \approx \int p(x) d x$, where $p(x)$ is the momentum of a particle with a mass of $M$, total energy $E$, and potential energy $V(\mathrm{x})$. The momentum of a particle is defined as $p(x)=\sqrt{2 M[E-V(x)]}=\hbar \beta^{27}$. So the approximation in Eq. (9) reduces to the following equation which is the adiabatic parameter for the $j$ th groove:

$$
\delta_{j}=\frac{\frac{1}{\beta_{j}}-\frac{1}{\beta_{j+1}}}{\Lambda_{j}}
$$

where $\wedge j=d+w_{j}$ and $w_{j}=w+j \Delta$ as shown in Fig. 1a. Please refer to the "Supplementary information S1" for a proof of Eq. (10). Figure 3 shows the adiabatic parameter (calculated within the grooves across the nano-grating structure) as a function of the period and the gradient in groove width. It is observed that larger values of the period and smaller values of the adiabatic parameter $\delta$ improve the adiabaticity between the grooves.

Single groove $(\mathbf{n}=\mathbf{1})$. In this section, we investigate the impact of the groove width $(w)$ on the resonance behaviour of a single groove carved in a metallic gold slab. The local optical inhomogeneity created by the single groove is not sufficient to match the in-plane $k$ vectors of the incident light with the SPPs. Thus, the field localization within a single groove seen in Fig. 4 is mostly driven by the photonic modes inside the cavity that directly couple to the incident light through an end-fire coupling mechanism ${ }^{28}$. Also, Fig. 4 shows that stepwise increase in $w$ from 10 to $20 \mathrm{~nm}$ correspondingly decreases the resonance wavelength $\left(\lambda_{\text {resonant }}\right)$ from $\sim 5.2$ to $\sim 3.5 \mu \mathrm{m}$ in the mid-infrared regime, or $\sim 1600$ to $\sim 1000 \mathrm{~nm}$ in the near-infrared regime.

Close examination of Fig. 4 indicates a counterintuitive inverse relationship between $w$ and $\lambda_{\text {resonant, }}$ in contrast to that in a Fabry-Perot cavity where larger grooves support longer plasmonic wavelengths; that is, the plasmonic resonant wavelength within the groove increases with decreasing groove width $(w)$. The underlying reason for this behaviour is the fact that the effective index of the groove increases as it narrows ${ }^{11}$. Hence, for a fixed groove depth the wavelength of the Fabry-Perot mode as supported by a narrower groove increases in accordance to Eq. (4).

At resonance, the incident wave, SPPs, and other surface waves on the metal surface couple to the photonic cavity mode leading to a high intensity electric field localized inside the groove. The localized surface plasmons reradiate into free space and dissipate in the surrounding metal within the cavity. However, at wavelengths below or above the resonance, the surface waves do not couple to the cavity modes and propagate away from the groove. Two regimes of operation can be realized based on the groove width in a grating structure: electrostatic regime $(\text { for } w<10 \mathrm{~nm} \text { ) and optical regime (for } w>10 \mathrm{~nm})^{17}$. For visible excitation frequencies, decreasing the width of the groove down to a few nanometers moves the dispersion of the guided mode within the grooves from the 


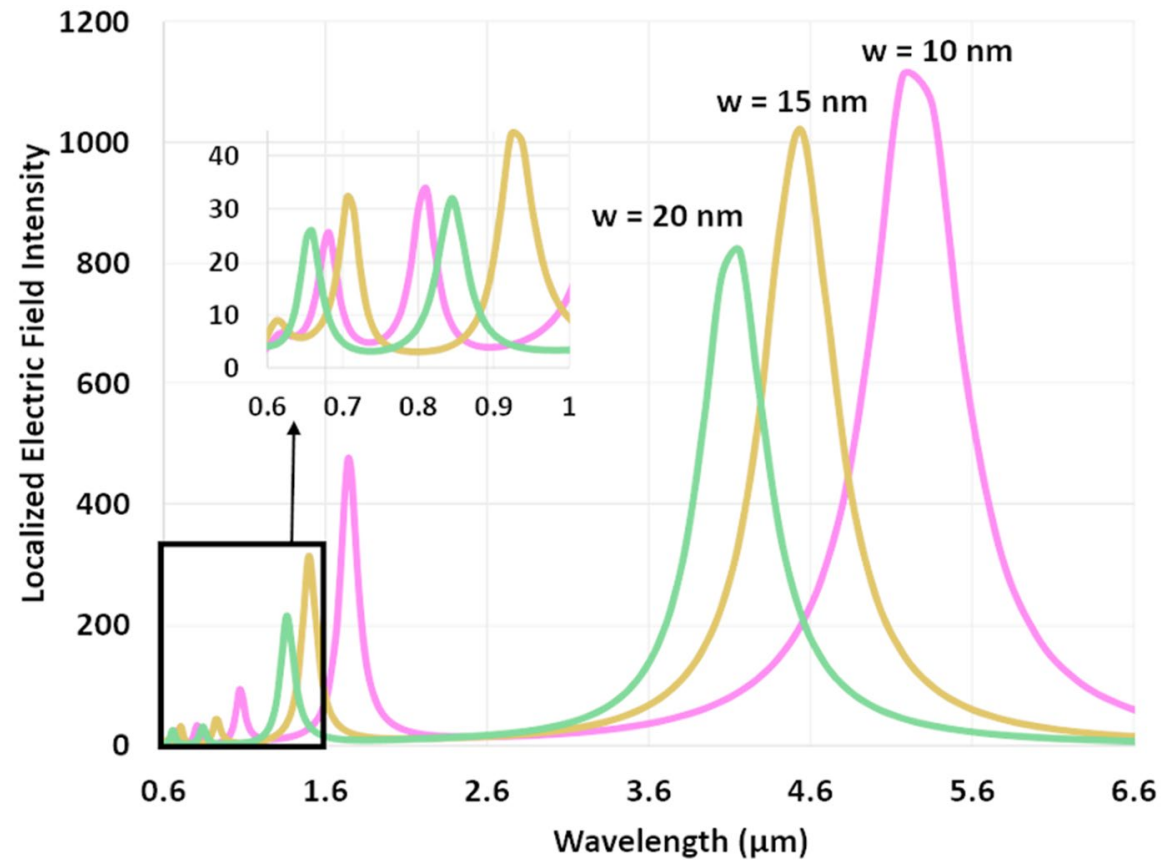

Figure 4. Resonance spectrum of a single groove for different groove widths $(w)$ and groove depth $(L)=500 \mathrm{~nm}$. Wavelength and localized intensity in the groove cavity increase as $w$ decreases.

optical to the electrostatic regime. For example, at $\lambda=500 \mathrm{~nm}$, the crossover point between these two regimes is a groove width of $10 \mathrm{~nm}^{17}$. Whereas at larger wavelengths in the infrared, the crossover point is at a smaller groove width. To carry out consistent numerical analyses, we performed the simulation in the optical regime where the minimum groove width is kept at $w=10 \mathrm{~nm}$.

Our simulation results also indicate that reducing the groove width reduces the full-width at half-maximum (FWHM) of the resonant peak of the groove and thus enhances the $Q$-factor. The $Q$ factor is calculated using $v_{\text {resonant }} /$ FWHM, where $v_{\text {resonant }}$ is the resonant frequency of the groove. In the mid-infrared regime, for simulated groove widths of $w=10,15$, and $20 \mathrm{~nm}$, FWHM (Q-factor) assume values of $2.9 \mu \mathrm{m}(1.6), 1.5 \mu \mathrm{m}(2.3)$, and $0.7 \mu \mathrm{m}$ (5.5), respectively. In the visible regime, these values change to $35 \mathrm{~nm}$ (19.5), $36 \mathrm{~nm}(19.4)$, and $37 \mathrm{~nm}$ (17.5) for groove widths of $w=10,15$, and $20 \mathrm{~nm}$, respectively. It is observed that in the mid-infrared regime, FWHM ( $Q$ factor) has a decreasing (increasing) trend with increasing groove width, while in the visible regime this trend changes to increasing (decreasing). Although, the $Q$ factors appear to be small, $Q$ factor values normalized to the volume $(Q / V)$ for such subwavelength gratings are comparable to those of conventional optical resonators $^{22}$. Please see the supplementary information for a comparison of the $Q / V$ values between our graded grating and those of optical resonators.

The theoretical data in Fig. 2 and the simulation results in Fig. 4 indicate that multiwavelength localization is possible via proximal repetition of subwavelength nano-grooves that have a gradient in their widths.

Multiple grooves with constant groove width $(n>1$ and $\Delta=0)$. We next simulate a uniform lamellar grating with a varying number of grooves to investigate the resonance profile, localized field intensity and FWHM of the grooves. The results are summarized in Table 1.

Proximal repetition of nano-grooves reinforces SPPs over the grating surface. Increasing the number of grooves $(n)$ introduces the groove-to-groove separation $(d)$ as a new degree of freedom and accordingly improves matching of the in-plane $k$ vectors of an incident wave and the SPPs. This strengthens SPPs on the top interface between the grating and air. When $d=5 \mathrm{~nm}$, the separation between the grooves is less than the skin depth of the metal $\left(\delta_{\text {metal }}\right)$, consequently inter-groove evanescent field coupling between the adjacent grooves plays a significant role in defining the resonance profile of the structure. In this case, the resonant fields tunnel through the metal, without necessarily coupling to the SPPs on the grating surface. This results in a lower intensity of the localized electric field within the grooves which in turn lowers the effective Q-factor (i.e., increases the FWHM), where the effective $Q$-factor of a grating structure is taken as the average of the individual $Q$-factors of the grooves within the given grating structure.

The SPPs propagating on the grating surface and other non-propagative SPPs resulting from the scattering of light at the groove edges can couple to the Fabry-Perot modes inside the grooves. For $d>\delta_{\text {metal }}$, the constructive interference between the SPPs on the grating surface and their coupling to the Fabry-Perot modes define the resonance profile of the structure. For example, at $d=300 \mathrm{~nm}$ and $700 \mathrm{~nm}$ such constructive interference leads to a high intensity of the localized field. Upon increasing $d$ further, the SPPs propagate farther on the metallic surface of the grating prior to reaching the next groove and accordingly they experience a decay in intensity due 


\begin{tabular}{|c|c|c|c|c|c|c|c|c|c|c|c|c|c|c|}
\hline \multirow{3}{*}{$\begin{array}{l}\boldsymbol{n} \\
j \\
d(\mathrm{~nm})\end{array}$} & \multirow{2}{*}{\multicolumn{2}{|c|}{\begin{tabular}{|l|}
$\mathbf{2}$ \\
0,1
\end{tabular}}} & \multicolumn{6}{|l|}{3} & \multicolumn{3}{|l|}{5} & \multicolumn{3}{|l|}{$\infty$} \\
\hline & & & $-1,1$ & 0 & $-1,1$ & 0 & $-1,1$ & 0 & $-2,2$ & $-1,1$ & 0 & - & - & - \\
\hline & 5 & 300 & \multicolumn{2}{|l|}{300} & \multicolumn{2}{|l|}{700} & \multicolumn{2}{|l|}{1000} & \multicolumn{3}{|l|}{300} & 5 & 300 & 700 \\
\hline$\lambda_{\text {resonant }}(\mathrm{nm})$ & 1016 & 1070 & 1153 & 1070 & \multicolumn{2}{|l|}{1110} & \multicolumn{2}{|l|}{1166} & 1131 & 1070 & $\begin{array}{l}1131 \\
1070\end{array}$ & 576 & 1070 & \begin{tabular}{|l|}
$(723)$ \\
1111
\end{tabular} \\
\hline Intensity & 65 & 224 & 501 & 523 & 925 & 1946 & 620 & 603 & 311 & 441 & $\begin{array}{l}695 \\
691\end{array}$ & 1.81 & 491 & $\begin{array}{l}(445) \\
1307\end{array}$ \\
\hline FWHM (nm) & 227 & 182 & 158 & 128 & 80 & 80 & 86 & 84 & 145 & 112 & 170 & 237 & 129 & $\begin{array}{l}1) \\
77\end{array}$ \\
\hline Effective $Q$ factor & 4.5 & 6 & \multicolumn{2}{|l|}{7.2} & \multicolumn{2}{|l|}{14} & \multicolumn{2}{|l|}{13.88} & \multicolumn{3}{|l|}{8} & 2.7 & 8.3 & \begin{tabular}{|l|}
$(723)$ \\
14.3
\end{tabular} \\
\hline
\end{tabular}

Table 1. Resonance wavelength, intensity, FWHM, and effective $Q$ factor for uniform lamellar gratings with $w=15 \mathrm{~nm}, \Delta=0, L=100 \mathrm{~nm}, n>1$. Note that bi-wavelength localization is achieved at $d=300 \mathrm{~nm}$ for $n=3$ and 5 , and $d=700 \mathrm{~nm}$ for a periodic structure, whereas at larger values of $d$ uni-wavelength localization is achieved.

to the ohmic losses. For example, at $d=1000 \mathrm{~nm}$ the Fabry-Perot modes inside the grooves only directly couple to the incident light. Table 1 shows that the latter coupling mechanism not only leads to a relatively lower localized electric field intensity but also a smaller effective $Q$-factor.

Identical groove depth, width, and groove-to-groove separation in a uniform grating implies that such a structure would only resonate at a single wavelength of light. However, Table 1 shows that this structure resonates at two different wavelengths at a groove depth of $L=100 \mathrm{~nm}$, and higher order modes are excited at deeper groove depth of $L=500 \mathrm{~nm}$, as shown in Fig. 5a. According to Eq. (5), the propagation constant of the SPPs coupling to the cavity modes (mechanism II) can be both positive or negative depending on its direction of propagation, which leads to localization of two different wavelengths.

As $d$ increases, the SPPs on the grating surface decay and the presence of bi-wavelength localization reduces to uni-wavelength localization in uniform gratings. For example, at $d=1000 \mathrm{~nm}$ and $n=3$ (see Table 1), all three grooves localize a single wavelength at $\lambda=1166 \mathrm{~nm}$, whereas at $d=300 \mathrm{~nm}$ bi-wavelength localization is achieved throughout the grating structure. Evidently, localization of more than one wavelength of light in a nano-grating structure is linked to the strength of the SPPs propagating atop the grating surface and their coupling to the cavity modes where available.

As $n$ increases to infinity, diffraction orders in a uniform lamellar grating begin to emerge for $d \geq \lambda$. Starting from $d \approx 600 \mathrm{~nm}$, every groove acts as a quasi-point source and higher order diffraction modes emerge. Described by the Huygens-Fresnel principle, the propagating diffracted wave is the superposition of the secondary wavelets created by these point sources. As a result, the structure is also a diffraction grating and the diffraction orders add to the propagation constant of the SPPs according to Eq. (4). The magnitude of the first order diffracted wave is at its maximum at $d=700 \mathrm{~nm}$. This effect gives rise to a very small FWHM of $1 \mathrm{~nm}$ and a very high $Q$-factor of 723 [i.e., at $n=\infty, d=700 \mathrm{~nm}$ and $\lambda_{\text {resonant }}=723 \mathrm{~nm}$ (see Table 1 for the resonant wavelengths which are given inside the parentheses)]. At larger values of $d$, with $n$ still at infinity, higher order diffracted modes also contribute, albeit insignificantly to the propagation constant of the SPPs and lead to low intensity higher order peaks which have larger FWHMs.

Graded gratings $(\Delta \neq 0)$. Next, we carry out a series of simulations to study the localized electric field profiles in the graded gratings. In these simulations, we set $w=10 \mathrm{~nm}$ and $\Delta=5 \mathrm{~nm}$, to stay within the optical regime, as mentioned in the section on single groove. To simplify the simulations, we considered a non-symmetric (one-sided) graded grating with $n=5$ and $j$ ranging from 0 to -4 . A one-sided graded grating is shown in the inset of Fig. 5b wherein width of the nano-grooves gradually increases only in one direction, namely, to the left of minimum width groove labelled $j=0$. In contrast in a two-sided or symmetric nano-grating, as shown in Fig. 1, the nano-groove widths gradually increase in size symmetrically, in both directions, about the centrally situated minimum groove width. The value of $L$ and $d$ are varied from $10 \mathrm{~nm}$ to $1 \mu \mathrm{m}$, and 5-10 $\mu \mathrm{m}$, respectively; we present the results for $L=500 \mathrm{~nm}$ and $d=300 \mathrm{~nm}$ (see Figs. 5b and 6) considering that adiabatic coupling is improved when $300 \mathrm{~nm} \leq \mathrm{d} \leq 900 \mathrm{~nm}$ (which is discussed further below). The field intensities shown in Fig. 5, similar to those in Fig. 1, are calculated within the nano-groove and normalized with respect to the incident field.

In a graded grating structure, the effective refractive index of the grooves increases with decreasing groove width; that is, a width-graded grating is also a structure graded in effective index. Thus, the group velocity of the SPPs traveling on the grating surface changes with the width of the groove and reaches a minimum across the narrowest groove. This variation in group velocity along with the constraint of conservation of energy gives rise to multiwavelength light localization in a width-graded grating as defined here (where $d$ is a constant).

The multiwavelength localization shown in Fig. $5 \mathrm{~b}$ is of an impedance-matched graded grating which benefits from adiabatic mode transformation. Such graded grating structures provide multiwavelength localization of light over a large bandwidth that stretches from approximately $600 \mathrm{~nm}$ to about $6 \mu \mathrm{m}$, in contrast to a uniform grating (Fig. 5a) which offers only uni-wavelength localization in discrete spectrum range.

Figure 3 demonstrates that larger values of the period lead to better adiabaticity between the grooves. Nevertheless, as noted from Table 1, intensity of the SPPs on the grating surface decay for $d>1 \mu \mathrm{m}$, due to ohmic losses. As a result, at $300 \mathrm{~nm} \leq d \leq 900 \mathrm{~nm}$ improved phase engineering of the SPPs leads to adiabatic mode transformation between adjacent grooves and thus leads to enhanced multiwavelength localization in the graded gratings 

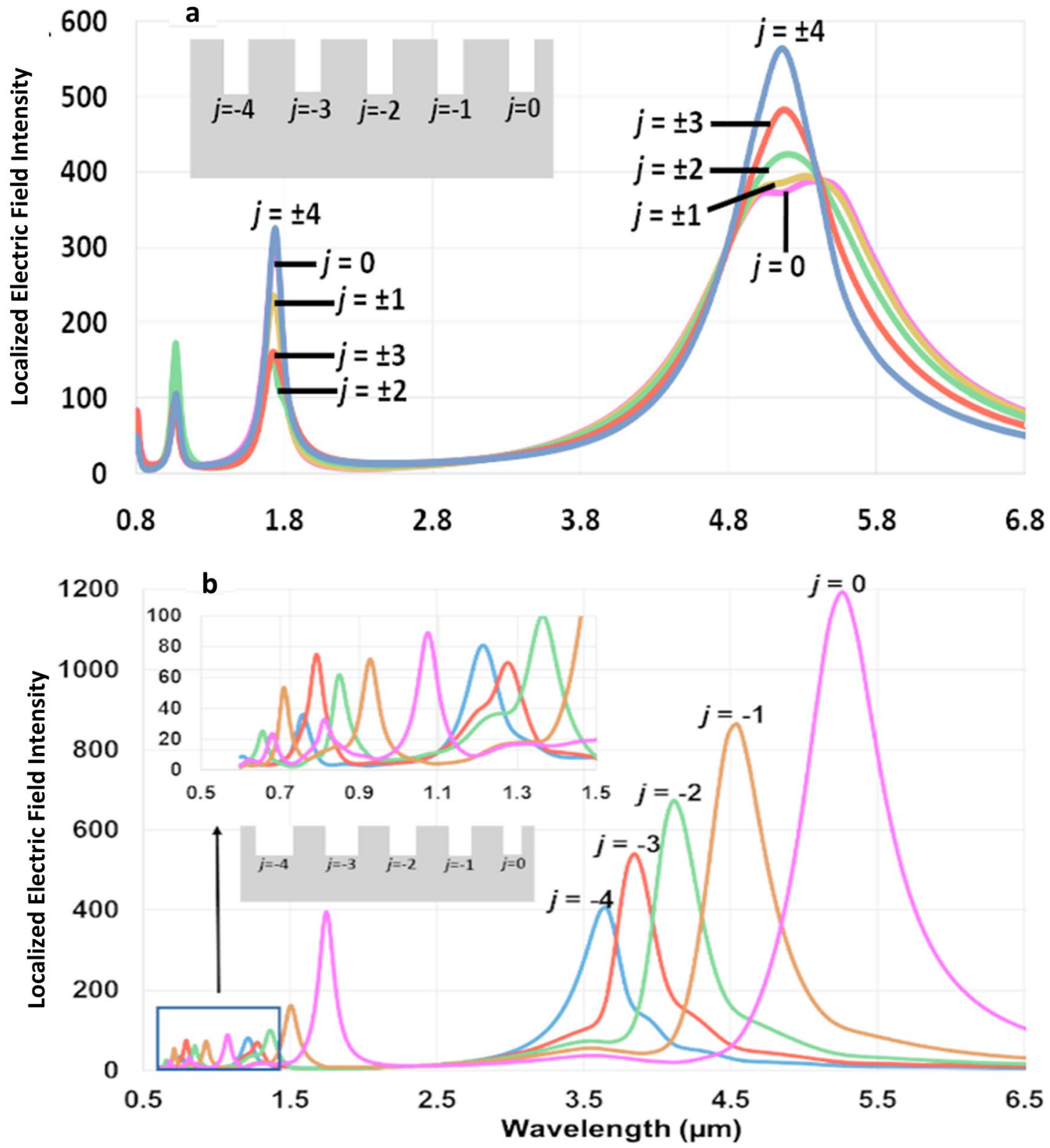

Figure 5. (a) Localized electric field intensity for a uniform grating with $L=500 \mathrm{~nm}, w=10 \mathrm{~nm}, n=9$ and $-4 \leq j \leq+4$. All the nano-grooves localize identical plasmonic modes, except around the first excited mode around $5 \mathrm{~mm}$ where small variations in the resonance mode of different grooves are noted, (b) localized electric field intensity for a non-symmetric graded grating with $L=500 \mathrm{~nm}, w=10 \mathrm{~nm}, \Delta=5 \mathrm{~nm}, n=5$ and $-4 \leq j \leq 0$. Every groove resonates at a particular wavelength of light, and impedance matched graded grating exhibits a multiwavelength mode localization in a wide bandwidth of $600 \mathrm{~nm}$ to $6 \mu \mathrm{m}$. This multiwavelength localization is resulted from adiabatic mode transformation.

(as shown in Fig. $5 \mathrm{~b}$ for $d=300 \mathrm{~nm}$ ). Under adiabatic mode transformation, the graded grating is impedancematched, that is, each groove resonates strongly at a single wavelength while transferring their non-resonant modes to an adjacent groove(s) so that the modes overlap with the resonant mode of the neighbouring groove(s).

Figure 6 shows how adjacent grooves couple and guide their non-resonant modes to the neighbouring resonating mode in an impedance-matched graded grating. The blue vectors pointing to the right (left) correspond to positive (negative) values of the real part of the $x$-component of the electric field $\left(\operatorname{Re}\left(\vec{E}_{x}\right)\right)$. The resonating groove, indicated on the figure, exhibits the highest field strength $\left(E=\left(\left|E_{x}\right|^{2}+\left|E_{y}\right|^{2}+\left|E_{z}\right|^{2}\right)^{1 / 2}\right)$ which evidently corresponds to the highest total power dissipated at the bottom of the groove. $\operatorname{Re}\left(\vec{E}_{x}\right)$ of the resonating groove points to the right while the field in all the other grooves point toward the resonating groove. For example, when groove $j=0$ (Fig. 6a) is at resonance (at $\left.\lambda_{\text {resonant }}=5.264 \mu \mathrm{m}\right), \operatorname{Re}\left(\vec{E}_{x}\right)$ of all the other grooves point towards it. Similarly, when groove $j=-1$ is at resonance (at $\left.\lambda_{\text {resonant }}=4.478 \mu \mathrm{m}\right), \operatorname{Re}\left(\vec{E}_{x}\right)$ of all the other grooves point 

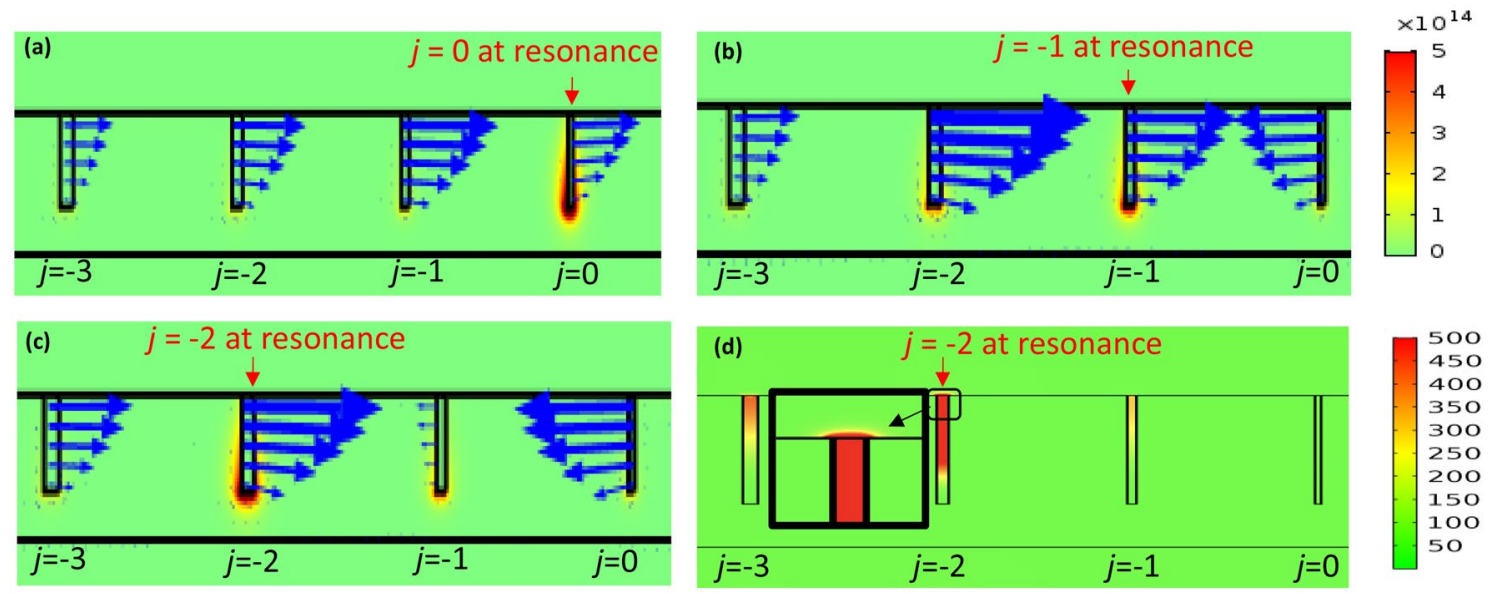

Figure 6. (a) $j=0$, (b) $j=-1$, and (c) $j=-2$ correspond to $\lambda_{\text {resonant }}=5.264 \mu \mathrm{m}, 4.478 \mu \mathrm{m}$, and $4.167 \mu \mathrm{m}$, respectively, where the groove under resonance is labelled/identified. Total power dissipation density, the rainbow colors surrounding each groove, shows the amount of dissipated electric field which is evidently highest at the bottom of the groove under resonance. The blue vectors show the direction of the scattered electric field, real part of $\vec{E}_{x}$, within each groove. These vectors in any groove point towards the groove under resonance and thus demonstrate how adiabatic mode transformation dictates that all non-resonating grooves direct their scattered field $\overrightarrow{\boldsymbol{E}}_{\boldsymbol{x}}$ towards the resonating groove. (d) Normalized field intensity map of the graded grating when groove $j=-2$ is under resonance at $4.167 \mu \mathrm{m}$. Note that the maximum field is localized within this groove in comparison with the rest. The inset in this figure shows the localized field and in particular the field extending a few nanometers $(\sim 10 \mathrm{~nm})$ above the nano-groove surface. The scale bar on the right of Fig. $6 \mathrm{~b}$ corresponds to $(\mathbf{a}-\mathbf{c})$ while the scale bar to the right of Fig. $6 \mathrm{~d}$ corresponds to $(\mathbf{d})$. Figures were obtained using COMSOL Multiphysics, version 5.3. COMSOL Inc, http://www.comsol.com.

toward the resonating groove. Our simulation results show that this adiabatic mode transformation occurs for every groove only when $300 \mathrm{~nm} \leq d \leq 900 \mathrm{~nm}$. This in turn leads to enhanced multiwavelength localization of light. Under these conditions of adiabaticity, the graded grating structure is said to be impedance matched such that the energy is efficiently transferred from the incident light to the SPPs on the surface of the grating and to the photonic modes of the grooves. For $50 \mathrm{~nm} \leq d<300 \mathrm{~nm}, \operatorname{Re}\left(\vec{E}_{x}\right)$ in the grating changes its direction multiple times above the resonant frequency and thus does not allow coupling of a single dominant plasmonic resonant mode to the grooves, thereby leading to poor multiwavelength localization. Please refer to supplementary information where Figs. S1a and S2a show profiles of the $\operatorname{Re}\left(\vec{E}_{x}\right)$ across visible and near-IR regions for $d=300 \mathrm{~nm}$ and $d=100 \mathrm{~nm}$, respectively, and Figs. S1b and S2b show corresponding variation of phase of $E_{\mathrm{x}}$. Also, see supplementary information Visualization 1 animating the behavior of $\operatorname{Re}\left(\vec{E}_{x}\right)$ and the resonance profile of the graded grating for $d=100 \mathrm{~nm}$ and $d=300 \mathrm{~nm}$, at $L=100 \mathrm{~nm}$.

Figure $6 \mathrm{~d}$ shows a plot of the normalized field intensity localized within the nano-grooves when $j=-2$ is under resonance. This figure represents the field map of the structure in Fig. $5 \mathrm{~b}$ at the resonant wavelength of groove $j=-2$ which is $\lambda_{\text {resonant }}=4.167 \mu \mathrm{m}$. The field localized within the groove is situated towards the top of cavity and extends about $10 \mathrm{~nm}$ above the resonating nano-groove surface. Please see the supplementary information Fig. S6 for a resonant field map within a lamellar uniform-width grating structure corresponding to the resonant profile shown in Fig. 5a. The reason for non-adiabatic behaviour of $\vec{E}_{x}$ for $d<300 \mathrm{~nm}$ is that the propagation constant of the SPPs on the grating surface is smaller than those resonating within the groove, thus preventing their coupling. However, under adiabatic mode transformation, for $300 \mathrm{~nm} \leq d \leq 900 \mathrm{~nm}$, the propagation constant of the SPPs on the grating surface is larger or equal to those resonating within the groove which allows for mode coupling in between the grooves, based on Eqs. (6) and (8).

Table 2 shows the resonance profile of a (non-symmetric) one-sided graded grating. The resonant wavelengths and intensities of this structure are similar to that of a symmetrically graded grating, except for the localized field intensity in the center groove $(j=0)$ of the symmetric grating which is twice as large as that in the non-symmetric grating. This result demonstrates that the graded grating not only exhibits multiwavelength light localization, but also serves as a waveguide to propagate the off-resonant modes from the outer-lying wider grooves toward the narrower central-lying grooves. At a groove depth of $L=100 \mathrm{~nm}$, only the first plasmonic mode is excited within the nano-grooves. However, deeper nano-grooves excite additional plasmonic modes and Table 2 shows that the nano-grooves support up to 5 plasmonic modes at $L=500 \mathrm{~nm}$. Evidently, the smallest groove $(j=0)$ supports additional localized modes compared to the neighboring wider nano-grooves. These numerical observations also corroborate with our theoretical calculations in Fig. 2 wherein the relationships between the wavelength of the localized field, groove depth, width and mode number are shown.

FWHM and $Q$ factor of the graded gratings for different values of $d$ is tabulated in Table 2. An optimized graded grating structure should possess a small FWHM, large $Q$ factor, and multiwavelength localization in all grooves. Based on Table 2, the FWHM and Q factor of a shallow graded grating with $L=100 \mathrm{~nm}$ ranges between 146 and $185 \mathrm{~nm}$ and 5.28-8.57, respectively. Whereas a deeper graded grating with $L=500 \mathrm{~nm}$ offers better values of FWHM and Q factor for higher order modes, albeit at the cost of reduced intensity for these localized 


\begin{tabular}{|c|c|c|c|c|c|c|c|c|c|c|}
\hline $\mathrm{J}$ & -4 & -3 & -2 & -1 & 0 & -4 & -3 & -2 & -1 & 0 \\
\hline $\mathrm{L}(\mathrm{nm})$ & \multicolumn{5}{|l|}{100} & \multicolumn{5}{|l|}{500} \\
\hline \multicolumn{11}{|c|}{$\lambda_{\text {resonant }}(\mu \mathrm{m})$} \\
\hline 1st mode & 0.92 & 0.952 & 1.07 & 1.13 & 1.25 & 3.66 & 3.85 & 4.11 & 4.55 & 5.26 \\
\hline 2nd mode & & & & & & 1.21 & 1.28 & 1.36 & 1.51 & 1.74 \\
\hline 3rd mode & & & & & & 0.75 & 0.79 & 0.85 & 0.93 & 1.08 \\
\hline 4th mode & & & & & & & & 0.65 & 0.71 & 0.811 \\
\hline 5th mode & & & & & & & & & & 0.68 \\
\hline \multicolumn{11}{|c|}{ Intensity $\times 10^{11}\left(\mathrm{~V}^{2} / \mathrm{m}^{2}\right)$} \\
\hline 1 st mode & 0.28 & 0.67 & 0.88 & 0.99 & 1.17 & 1.13 & 1.51 & 1.89 & 2.42 & 3.34 \\
\hline 2nd mode & & & & & & 0.226 & 0.195 & 0.28 & 0.45 & 1.11 \\
\hline 3rd mode & & & & & & 0.1 & 0.21 & 0.17 & 0.2 & 0.25 \\
\hline 4th mode & & & & & & & & 0.07 & 0.15 & 0.09 \\
\hline 5th mode & & & & & & & & & & 0.06 \\
\hline \multicolumn{11}{|c|}{ FWHM (nm) } \\
\hline 1 st mode & 146 & 165 & 183 & 185 & 153 & 380 & 325 & 343 & 477 & 649 \\
\hline 2nd mode & & & & & & 86.3 & 139 & 105 & 115 & 130 \\
\hline 3rd mode & & & & & & 43 & 49.1 & 55.2 & 54.5 & 65.1 \\
\hline 4th mode & & & & & & & & 34.3 & 32.5 & 60.4 \\
\hline 5th mode & & & & & & & & & & 33.9 \\
\hline \multicolumn{11}{|l|}{$Q$ factor } \\
\hline 1st mode & 6.3 & 5.73 & 5.28 & 6 & 8.57 & 9.32 & 11.9 & 12.2 & 9.57 & 8.14 \\
\hline 2nd mode & & & & & & 14 & 8.89 & 12.9 & 13.1 & 13.4 \\
\hline 3rd mode & & & & & & 17.4 & 16.1 & 15.6 & 17 & 16.5 \\
\hline 4th mode & & & & & & & & 19.1 & 21.9 & 13.7 \\
\hline 5th mode & & & & & & & & & & 20 \\
\hline
\end{tabular}

Table 2. Resonance wavelength, intensity, FWHM, and effective $Q$ factor for a non-symmetric graded grating with $w=10 \mathrm{~nm}, \Delta=5 \mathrm{~nm}, d=300 \mathrm{~nm}, n=5$. Note that increasing the groove depth from 100 to $500 \mathrm{~nm}$ increases the range of the localized wavelength across a larger spectral range extending from the visible to the mid-infrared.

modes. Our simulation results also indicate that using other dielectrics such as $\mathrm{SiO}_{2}$ instead of air in the MIM grooves introduces a higher index contrast and lowers the average FWHM at the cost of the localized intensity. For example, using $\mathrm{SiO}_{2}$ as an insulator in the MIM grooves at $L=100 \mathrm{~nm}$ yields a FWHM of $169 \mathrm{~nm}$, an effective $Q$ factor of 9.2, and an intensity of $0.87 \times 10^{10}\left(\mathrm{~V}^{2} / \mathrm{m}^{2}\right)$ for $j=-1$.

Our simulation results also reveal that contrary to the red-shifting of the resonant wavelength in uniform gratings with increasing $d$, in the width-graded grating the resonant wavelength of the grooves neither redshifts nor blueshifts with changing $d$. Accordingly, the resonant wavelength(s) of each groove in an adiabatically coupled graded grating structure is simply determined by examining the resonant wavelength(s) of the corresponding single groove. Inspecting Figs. 4 , 5b, and Table 2, we observe that identical resonant wavelengths are realized for grooves with identical groove width in both the single groove structure and the graded grating structure. However, determination of the intensity and FWHM for a resonant mode in a graded grating requires modeling of the grating structure as a whole. These results corroborate a previous study wherein a simple model for a single groove was used to explain the behavior of an entire functionally graded grating with non-homogenous effective index 9 .

A graded grating structure with the resonant profile shown in Fig. 5a offers enhanced rainbow trapping in the visible, near-infrared, and mid-infrared spectral ranges with many potential benefits for surface enhanced Raman, fluorescence, and infrared spectroscopy, and hence sensing applications, by virtue of overlapping vibrational modes of interest ${ }^{1-3,29,30}$. Further, fabrication of such high aspect ratio structures is feasible via precise lithographic techniques ${ }^{31}$, facile multi-layer sputter-deposition of thin metal-dielectric layers ${ }^{20}$, tapered-side wall mold technique ${ }^{32}$, or mold cast techniques based on electrolytic growth of gold ${ }^{33}$.

\section{Conclusion}

We have identified four different coupling mechanisms that underpin the plasmonic resonance profile of subwavelength uniform and width-graded lamellar gratings in the visible and near-mid infrared regions. We have utilized this framework to develop unique insight into the underlying physics of adiabatic mode transformation between the nanogrooves which leads to multiwavelength light localization in a width-graded grating. The simulation results for uniform and width-graded gratings reveal that inter-groove evanescent field coupling of adjacent grooves (with groove-to-groove separations of less than $50 \mathrm{~nm}$ ) lowers the $Q$ factor of the structure which in turn leads to a diffusion of the resonant profiles over all the grooves-groove hybridization-and hence impairs multiwavelength localization. However, width-graded gratings with groove-to-groove separation of between 300 
and $900 \mathrm{~nm}$ yield an impedance matched graded grating structure as a result of improved adiabatic mode transformation between adjacent grooves, thus enhancing multiwavelength light localization. This optical adiabaticity among the grooves is most notably established when non-resonant modes of each groove are only transferred to the resonant groove. In such an impedance matched width-graded grating structure, the resonant wavelength is simply determined by examining the resonant wavelengths of the corresponding single groove. At these values of $d$, increasing the groove depth introduces additional resonant modes and thus extends multiwavelength light localization into the near and mid infrared, with improved FWHM and Q factor values for higher order modes in the visible region. This investigation shows that width-based graded gratings can provide adiabatic coupling of multiple wavelengths of light to the resonant modes of the graded grating and thus offers multiwavelength field intensification with eleven orders of magnitude relative to the incident field intensity. These structures which can be reproducibly and economically manufactured open the way for a manifold of high-field intensity sensing applications wherein molecular species of interest on a given width-graded nano-grating surface can be probed essentially simultaneously using several wavelengths spanning from the visible into the infrared and thus lead to high-specificity and high-sensitivity detection.

\section{Methods}

We used COMSOL Multiphysics modelling to study the near-field optical response of MIM graded grating structures in gold with air-filled cavities. The structure of the gratings is illustrated in Fig. 1a where $w$ is the width of the smallest groove with groove-index $j=0 . \Delta$ is the groove width gradient parameter, where $\Delta=0$ defines a uniform lamellar grating while $\Delta \neq 0$ defines a graded lamellar grating wherein the width of each groove increases linearly in increments of $\Delta$ in the $\pm x$ directions. Groove index values spanning $-4 \leq j \leq+4$ and $\Delta \neq 0$ thus create a symmetric graded grating structure around $j=0$. In our simulations, we chose the largest central groove width to be $30 \mathrm{~nm}$, with the gradient parameter $\Delta=5 \mathrm{~nm}$, in order to achieve strong evanescent field coupling between the side-walls of the MIM grooves and thus resulting in relatively flat dispersion curves in contrast to a single interface dispersion curve ${ }^{22}$. Further, we omit the use of a substrate in our simulations considering that the thickness of the metal beneath the grooves is $300 \mathrm{~nm}$ which is far greater than the skin depth. All simulations were executed spanning the visible and the near infrared wavelengths, $500 \mathrm{~nm}<\lambda_{\text {incident }}<6500 \mathrm{~nm}$. A direct solver (MUMPS) was used to solve Maxwell's equations in the frequency domain. The electric field $E=\left(\left|E_{x}\right|^{2}+\left|E_{y}\right|^{2}+\left|E_{z}\right|^{2}\right)^{1 / 2}$ was measured within each groove, and it was squared to obtain the localized electric field intensity. All the simulations were based on a 2D model where the groove length in the $y$ direction (into the page in Fig. 1) was assumed to be infinite considering that groove lengths greater than $1 \mu \mathrm{m}$ do not significantly alter the resonance behaviour of the structure ${ }^{16}$ in the near/mid infrared frequency region and even less so in the visible. The refractive index of gold was obtained from references ${ }^{34,35}$. All modelled structures were illuminated by normally incident (from the top) TM plane waves. A perfectly matched layer boundary condition was used to avoid contributions from any scattered light at the boundary of the modelled structure. For the case of uniform lamellar grating with an infinite number of grooves, where the repeating unit is one single groove, we used the Floquet periodic boundary condition on both sides of the unit groove. This periodic boundary condition accounted for diffracted orders of the incident light for grating periods larger than the incident light wavelength.

Received: 20 April 2020; Accepted: 30 November 2020

Published online: 12 January 2021

\section{References}

1. Roelli, P., Galland, C., Piro, N. \& Kippenberg, T. J. Molecular cavity optomechanics as a theory of plasmon-enhanced Raman scattering. Nat. Nanotechnol. 11, 164-169 (2015).

2. Kazemi-Zanjani, N. et al. Multiwavelength surface-enhanced Raman spectroscopy using rainbow trapping in width-graded plasmonic gratings. Adv. Opt. Mater. 6(4), 1-8 (2018).

3. Luan, J. et al. Add-on plasmonic path as a universal fluorescence enhancer. Light Sci. Appl. 7(29), 1-13 (2018).

4. Chen, B. et al. Enhanced fluorescenec for in situ temperature mapping of photothermally heated aluminum nanoparticles enabled by a plasmonic grating substrate. Nanotechnology 29, 1-10 (2018).

5. Han, C., Lee, M., Callard, S., Seassal, C. \& Jeon, H. Lasing at topoligical edge states in a photonic crystal L3 nanocavity dimer array. Light Sci. Appl. 8(40), 1-10 (2019).

6. Najiminaini, M., Vasefi, F., Kaminska, B. \& Carson, A. J. J. L. A three-dimentional plasmonic nanostructure with extraordinary optical transmission. J. Plasmonics 8(2), 217-224 (2013).

7. Ikeda, K. et al. Controlled thermal emission of polarized infrared waves from arrayed plasmon nanocavities. Appl. Phys. Lett. 92, 021117 (2008).

8. Miyazaki, H. T. et al. Thermal emission of two-color polarized infrared waves from integrated plasmon cavities. Appl. Phys. Lett. 92, 141114 (2008).

9. Jiang, L. et al. In-place coherent control of plasmon resonances for plasmonic switching and encoding. Light Sci. Appl. 8(21), 1-10 (2019).

10. Nishijima, Y., Rosa, L. \& Juodkazis, A. S. Surface plasmon resonances in periodic and random patterns of gold nano-disks for broadband light harvesting. Opt. Express 20(10), 11466-11477 (2012).

11. Montazeri, A. O., Fang, Y. S., Sarrafi, P. \& Kherani, N. P. Rainbow-trapping by adiabatic tuning of intragroove plasmon coupling. Opt. Express 24(23), 26745-26755 (2016).

12. Polyakov, A., Zolotorev, M., Schuck, P. J. \& Padmore, H. A. Collective behavior of impedance matched plasmonic nanocavities. Opt. Express 20(7), 7685-7693 (2012).

13. Zhu, L. et al. Dynamically generating a large-area confined optical field with subwavelength feature size. Optical Soc. Am. 53(26), 6091-6095 (2014).

14. Verslegers, L. et al. Planar lenses based on nanoscale slit arrays in a metallic flim. Am. Chem. Society 9(1), 235-238 (2009). 
15. Manceau, J. M., Zanotto, S., Sagnes, I., Beaudoin, G. \& Colombelli, R. Optical critical coupling into highly confining metalinsulator-metal resonators. Appl. Phys. Lett. 103, 091110 (2013).

16. Collin, S., Pardo, F. \& Pelouard, A.J.-L. Waveguiding in nanoscale metallic apertures. Opt. Express 15(7), 4310-4320 (2007).

17. Perchec, J. L., Quemerais, P., Barbara, A. \& Lopez-Rios, A. T. Why metallic surfaces with grooves a few nanometers deep and wide may strongly absorb visible light. Phys. Rev. Lett. 100(6), 066408 (2008).

18. Gan, Q. \& Bartoli, F. J. Graded metallic gratings for ultrawideband surface wave trapping at THz frequencies. IEE J. Select. Topics Quant. Electron. 17(1), 102-109 (2011).

19. Tanenbaum, D. M., Lo, C. W., Isaacson, M. \& Craighead, H. G. High resolution electron beam lithography using ZEP-520 and KRS resists at low voltage. J. Vac. Sci. Technol. B 14(6), 3829-3833 (1996).

20. Huang, L. C., Wang, Z., Clark, J. K., Ho, Y. L. \& Delaunay, J. J. Plasmonic tooth-multilayer structure with high enhancement field for surface enhanced Raman spectroscopy. Nanotechnology 28(12), 1-7 (2017).

21. Yariv, A. \& Yeh, P. Photonics-Optical Electronics in Modern Communications (Oxford University Press, New York, 2007).

22. Maier, S. A. Plasmonics: Fundamentals and Applications (Springer, New York, 2007).

23. Astilean, A., Lalanne, P. \& Palamaru, M. Light transmission through metallic channels much smaller that the wavelength. Optics Commun. 17, 265-273 (2000).

24. Han, Z., Forsberg, E. \& He, S. Surface plasmon Bragg gratings formed in metal-insulator-metal waveguides. IEEE Photonics Technol. Lett. 19(2), 91-93 (2007).

25. H. T. Miyazaki \& A. Y. Kurokawa. Squeezing visible light waves into a 3-nm thick and 55-nm long plamson cavity. Phys. Rev. Lett. 96(9) (2006).

26. Griffiths, D. J. Introduction to Quantum Mechanics 2nd edn. (Pearson Education Inc., New Jersey, 2005).

27. Landau, L. D. \& Lifshitz, E. M. Quantum Mechanics-Non-Relativistic Theory (Pergamon Press, London, 1962).

28. Ceglia, D. D., Vincenti, M. A., Scalora, M. \& Bloemer, N. A. A. M. J. Plasmonic band edge effects on the transmission properties of metal gratings. AIP Adv. 1, 032151 (2011).

29. Neubrech, F., Huck, C., Weber, K., Pucci, A. \& Giessen, H. Surface-enhanced infrared spectroscopy using resonant nanoantennas. Chem. Rev. 117, 5110-5145 (2017).

30. Yang, X. et al. Nanomaterial-based plasmon enhanced infrared spectroscopy. Adv. Mater. 30, 1-30 (2018).

31. Polyakov, A. et al. Light trapping in plasmonic nanocavities on metal surfaces. J. Vac. Sci. Technol., B 29(6), 1-4 (2011).

32. Bouchon, P. et al. Total funneling of light in high aspect ratio plasmonic nanoresonators. Appl. Phys. Lett. 98, 191109 (2011).

33. Ogawa, S. \& Kimata, M. Direct fabrication and characterization of high-aspect-ratio plasmonic nanogratings using tapered-sidewall molds. Opt. Mater. Express 7(2), 633-640 (2017).

34. Christy, R. W. \& Johnson, P. B. Optical constants of the Noble Metals. Phys. Rev. B 6(12), 4370-4379 (1972).

35. Rakic, A. D., Djurisic, A. B., Elazar, J. M. \& Majewski, M. L. Optical properties of metallic films for vertical-cavity optoelectronic devices. Appl. Opt. 37(22), 5271-5283 (1998).

\section{Acknowledgements}

We gratefully acknowledge the support of the Natural Sciences and Engineering Research Council (NSERC) of Canada through the Discovery Grant and Postgraduate Scholarship, the Ontario Research Foundation-Research Excellence program, the Connaught Global Challenge Fund at the University of Toronto (UofT), EMH (Engineering Medicine Health) Seed program at UofT, the Department of Electrical \& Computer Engineering at Uof T, the Walter Sumner Foundation, and CMC Microsystems.

\section{Author contributions}

M.S. carried out the theoretical analyses and COMSOL computational modeling, and authored the first manuscript. A.M. contributed to the analyses of the results and reviewed the manuscript, K.D. carried out the adiabatic theory development, R.P. reviewed the manuscript with an emphasis on its introduction, and N.K.-Z. reviewed the manuscript. N.P.K. as project PI supervised the research and reviewed the article and analyses.

\section{Funding}

This study was funded by Ontario Research Foundation (ORF-RE09-016).

\section{Competing interests}

The authors declare no competing interests.

\section{Additional information}

Supplementary Information The online version contains supplementary material available at https://doi. org/10.1038/s41598-020-79815-9.

Correspondence and requests for materials should be addressed to N.P.K.

Reprints and permissions information is available at www.nature.com/reprints.

Publisher's note Springer Nature remains neutral with regard to jurisdictional claims in published maps and institutional affiliations.

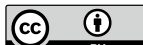

Open Access This article is licensed under a Creative Commons Attribution 4.0 International License, which permits use, sharing, adaptation, distribution and reproduction in any medium or format, as long as you give appropriate credit to the original author(s) and the source, provide a link to the Creative Commons licence, and indicate if changes were made. The images or other third party material in this article are included in the article's Creative Commons licence, unless indicated otherwise in a credit line to the material. If material is not included in the article's Creative Commons licence and your intended use is not permitted by statutory regulation or exceeds the permitted use, you will need to obtain permission directly from the copyright holder. To view a copy of this licence, visit http://creativecommons.org/licenses/by/4.0/.

(c) The Author(s) 2021 